\title{
SISTEMA DE DISTRIBUIÇÃO DE MEDICAMENTOS POR DOSE UNITÁRIA: REFLEXÕES PARA A PRÁTICA DA ENFERMAGEM
}

Jorséli Angela Henriques Coimbra* Elizabeth Amancio S.de S. Valsechi** Maria Dalva de Barros Carvalho*** Sandra Marisa Pelloso***

COIMBRA, J.A.H.; VALSECHI, E.A.S.de S.; CARVALHO, M.D.de B.; PELLOSO, S.M. Sistema de distribuição de medicamentos por dose unitária: reflexões para a prática da enfermagem. Rev.latino-am.enfermagem, Ribeirão Preto, v. 6, n. 4, p. 15-19, outubro 1998.

O enfermeiro, como parte integrante da equipe de saúde, está obrigado a conhecer a responsabilidade que tem na administração de medicamentos como algo importante dentro do conjunto de atividades que realiza. A implantação do sistema de distribuição de medicamentos por dose unitária se reflete diretamente nas atividades dos enfermeiros. Portanto, o objetivo deste trabalho é levantar pontos para reflexão com relação a esse sistema e suas repercussões na prática da enfermagem. Questiona-se a forma como o enfermeiro e sua equipe estão inseridos neste contexto de mudanças, no sentido de que realmente assegure o beneficio para o paciente, acompanhando e avaliando todo o processo, já que esta mudança afeta diretamente uma parte de sua atividade.

UNITERMOS: dose unitária, assistência de enfermagem

A enfermeira, como parte da equipe de saúde, está obrigada a conhecer a responsabilidade que tem na administração de medicamentos como algo importante dentro do conjunto de atividades que realiza. (PINERO et al., 1986, p. 107).

O decreto lei $\mathrm{n}^{\circ} 94.406$ de 1987 que regulamenta a lei $\mathrm{n}^{\mathrm{o}} 7.498$ que dispõe sobre o exercício profissional, em seu artigo $8^{\circ}$, relata as incumbências do enfermeiro, destacando entre outras: o planejamento, organização, coordenação, execução e avaliação dos serviços de assistências de enfermagem e a prescrição da assistência de enfermagem.

Sendo a medicação uma parte integrante e fundamental da assistência de enfermagem, pode-se inferir a responsabilidade do enfermeiro em relação a este aspecto. Esta responsabilidade é, mais uma vez, destacada no código de ética dos profissionais de enfermagem, no capítulo V - Das Proibições - artigo 47 dispondo que é proibido ao enfermeiro administrar medicamentos sem certificar-se da natureza das drogas que o compõem e da existência de risco para o paciente.

Tradicionalmente, a equipe de enfermagem é responsável pelo preparo e administração dos medicamentos aos pacientes hospitalizados. Com o avanço tecnológico, o aumento de serviços nas instituições hospitalares e sua diversificação, tornou-se mais intrincada a forma de distribuição de medicamentos nestas instituições.

Ajustar essa atividade de preparo e administração de medicamentos, uma das mais tradicionais e conservadoras atividades de enfermagem, às novas tendências globalizadoras se torna uma necessidade emergente da profissão. Por essa razão, considera-se que este trabalho possa contribuir na adequação da prática de enfermagem às mudanças que estão acontecendo.

Segundo MAIA NETO (1990, p. 28) "um sistema de distribuição envolve compras, controle de estoque, armazenamento e controle de qualidade, pessoal e uma série de qualidade do mesmo".

Para Garrison apud MAIA NETO (1990, p. 85) existem quatro tipos de distribuição de medicamentos: distribuição coletiva, individual, semi- individual e dose unitária.

A distribuição coletiva tem como característica

\footnotetext{
* Enfermeira. Professora Auxiliar do Departamento de Enfermagem da Universidade Estadual de Maringá

** Enfermeira. Professora Assistente do Departamento de Enfermagem da Universidade Estadual de Maringá

*** Enfermeira. Professora Adjunto do Departamento de Enfermagem da Universidade Estadual de Maringá

*** Enfermeira. Professora Adjunto do Departamento de Enfermagem da Universidade Estadual de Maringá
} 
o envio de uma certa quantidade de medicamentos para serem estocados nos setores e administrados conforme forem sendo prescritos. $\mathrm{O}$ segundo tipo caracteriza-se por serem os medicamentos distribuídos a cada paciente, ou seja, são encaminhadas medicações individuais, conforme a prescrição médica. O outro tipo, a forma semiindividual, é uma combinação dos dois tipos anteriores: é a distribuição individual mediante a prescrição médica, mas com um percentual de estocagem de medicamentos que permanecem nos setores.

O último tipo é o Sistema de Distribuição de Medicamentos por Dose Unitária - SDMDU, em que a medicação é preparada, identificada para a administração na posologia prescrita pelo médico, a partir da cópia da prescrição original, e encaminhada aos setores para administração.

O que diferencia este sistema dos outros é que, neste caso, os medicamentos são acomodados em embalagens unitárias, com horários e identificações, já prontas para a administração.

Justifica-se a implantação deste sistema, entre outros, por proporcionar a diminuição de erros no preparo da medicação, otimizar o tempo do pessoal de enfermagem, traduzidos pela elevação da qualidade de assistência prestada aos pacientes, e também por facilitar maior coesão do farmacêutico com a equipe de saúde.

É neste contexto de avanço que a enfermagem deve encaixar-se, bem como refletir acerca da distribuição de medicamentos por dose unitária. Esta modalidade de distribuição de medicamentos tende a se expandir cada vez mais.

Questiona-se então a forma como o enfermeiro e sua equipe estão inseridos neste contexto de mudança e implantação do sistema de distribuição de medicamento por dose unitária.

Se por um lado as modificações que asseguram o benefício para o expoente máximo do hospital, isto é, o paciente, são bem-vindas, por outro lado elas também merecem reflexões e proposições que assegurem este fazer com qualidade.

Que o enfermeiro não seja um mero seguidor de tendências, mesmo que basicamente inovadoras, mas que busque refletir sua prática, e que haja engajamento e comprometimento profissional.

Acreditamos que somente um trabalho coeso entre os elementos da equipe de saúde, alicerçado em estudos e reflexões, assegure uma implantação eficaz do sistema, com um olhar único para nossa finalidade máxima: o paciente.

Este trabalho busca refletir sobre o sistema de distribuição de medicamentos por dose unitária, tendo em vista que este tipo de distribuição está intimamente ligado às atividades dos profissionais de enfermagem.

Como intuito de bem compreender esta problemática, foi realizado um levantamento bibliográfico abrangendo os princípios, normas e rotinas da implantação do sistema e das experiências já realizadas.

$\mathrm{Na}$ literatura obtida abrangendo o período de 1981-1996 através da base LILACS (Literatura Latino Americana e do Caribe em Ciências da Saúde), resgatouse artigos sobre SDMDU elaborados por profissionais da área de farmácia. Estes descrevem normas e rotinas de implantação do SDMDU.

Apenas um artigo tendo como autora uma enfermeira, foi localizado na pesquisa bibliográfica. Neste, PINERO et al. (1986) analisa o sistema tradicional e o SDMDU apontando os benefícios deste último.

Tem-se relatos de algumas experiências vivênciadas, entre as quais pode se citar o Hospital e Maternidade Alvorada, unidade de Santo Amaro e Moema. Nestas, a implantação do SDMDU teve início em 1995. Os resultados obtidos apontam para uma redução de gastos na ordem de 20,10\%. Este dado foi encontrado comparando o ano de 1994, antes da implantação do SDMDU e o ano de 1995, quando se iniciou o novo sistema. (SANTOS, 1996, p. 02).

Nestas unidades o SDMDU foi implementado apenas para os medicamentos sólidos e líquidos, excetuando-se os via parentérico.

No Hospital Infantil Cândido Fontoura (São Paulo), o SDMDU foi implantado para todos os tipos de medicamentos a partir de 1996. Na avaliação de sua implantação os dados obtidos indicam redução de custos, analisados nos meses de maio (41,8\%); junho $(54,9 \%)$ e julho $(35,4 \%)$. Ainda foram avaliados os erros de preparação ocorridos no novo sistema com um percentual de 0,05\%. (MENDONÇA, 1996, p. 02).

Nos levantamentos não se encontrou referência em relação à integração da equipe de saúde e outras vantagens, sendo que todos mencionam como grande virtude do SDMDU a redução de custos.

Este rever bibliográfico denota quão o profissional enfermeiro está afastado do cerne de discussão tão importante, justificando assim, algumas reflexões.

\section{ALGUMAS REFLEXÕES}

Como em todos os casos de propostas para implantações de mudanças, são relatados benefícios que as justificam. Entre as várias vantagens citadas na SDMDU, vale ressaltar a "diminuição do tempo utilizado pelo pessoal de enfermagem para armazenagem e preparo de medicamentos com a conseqüente elevação da qualidade assistencial". Destaque-se ainda a diminuição na incidência de erros, economia de custos, aumento da segurança do profissional médico na garantia da utilização 
do medicamento certo, na dose certa e hora certa, segundo a prescrição médica, e aumento da integração do farmacêutico com os outros profissionais da equipe de saúde.

"A implantação de procedimentos racionalizados, como é o caso do SDMDU, vem trazendo benefícios consideráveis, seja na administração de medicamentos, na planilha de custos dos remédios e a própria prevenção das infecções hospitalares." (CONTROLE DE INFECÇÃO, 1996, p. 01).

Acreditamos que para adoção de qualquer medida renovadora é importante lembrar que os profissionais envolvidos devem estar preparados para as mudanças. Segundo a Organização Panamericana de Saúde, estudos relacionam os sistemas tradicionais com uma elevada incidência de erros. Quando se afirma que com a implantação do SDMDU ocorrerá a diminuição de erros no preparo da medicação, algumas reflexões se impõem. Tradicionalmente o preparo de medicamentos é realizado pelo auxiliar de enfermagem sob a supervisão do enfermeiro. $\mathrm{O}$ auxiliar de enfermagem tem formação teórico-prática e esta profissão é regulamentada no decreto-lei $\mathrm{n}^{\circ} 94.406$ de 08 de junho de 1987. Por sua formação, o auxiliar está habilitado para o preparo e administração de medicamentos e como profissional responde legalmente por seus atos. No SDMDU, MAIA NETO (1990, p. 103), ao descrever a rotina operacional da dose unitária, explicita que a função de preparo de medicamentos é do auxiliar de farmácia sob supervisão do farmacêutico. O auxiliar de farmácia, embora seja um prático treinado e supervisionado, não tem uma profissão regulamentada por lei e portanto passível de sanções.

Assim sendo, acreditamos que seria temeroso afirmar que a simples implantação do SDMDU diminuiria drasticamente os erros de medicações . É aceitável crer que isto possa ocorrer, porém alguns aspectos precisam ser analisados.

O enfermeiro ou auxiliar de enfermagem prepara e administra medicamentos na e para a sua unidade de atuação. O farmacêutico ou auxiliar de farmácia, no SDMDU prepara medicamentos para todo o hospital em clínicas diversas e setores extremamente específicos e complexos. Esta disparidade numérica no preparo de medicamentos, por si só pode ser fator de maior probabilidade de erros.

Para que efetivamente a diminuição de erros ocorra é necessário investir em recursos físico - estruturais e principalmente em recursos humanos, possibilitando um campo maior de atuação aos farmacêuticos no âmbito hospitalar.

O sistema de saúde no Brasil para o atendimento em nível hospitalar, compra serviços de terceiros, ou seja, de hospitais privados. A questão de custo-benefício do SDMDU é sempre bem-vinda para os empresários de saúde. Porém , o investimento para a adoção deste sistema é grande e é considerado pelo Ministério da Saúde como uma desvantagem da implantação do sistema, já que é preciso um grande investimento em recursos físicos e humanos, condizentes com um mínimo necessário para uma assistência com qualidade (BRASIL,1994, p. 57). Neste sentido é de fundamental importância a participação do enfermeiro junto ao farmacêutico quando da implantação do SDMDU no sentido de exigir infraestrutura e recursos, já que ele é o profissional que vai usufruir as benesses ou sofrer as distorções do sistema, enquanto membro da equipe de saúde.

Entendemos que os custos necessários para atender às exigências de infra-estrutura, de acordo com o decreto $\mathrm{n}^{\mathrm{o}} 8.015$ de julho de 1976, e de aumento dos recursos humanos são irrelevantes se observarmos o objetivo maior que é a melhoria da qualidade da assistência aos pacientes. Da mesma forma, devemos acrescentar que uma estrutura de estoque e de controle de medicamentos proposta na SDMDU proporcionaria redução dos desvios e das perdas relativas devido à caducidade e falta de identificação dos mesmos, que são comuns no modo tradicional. Entendemos que a redução de mão-de-obra manuseando o preparo das medicações oferece maior facilidade à supervisão, orientação e educação continuada, diminuindo assim o desperdício no preparo e na conservação das mesmas.

Com relação à maior integração do profissional farmacêutico junto à equipe de saúde no SDMDU é importante ressaltar que esta relação só acontece quando existe conhecimento científico de um todo relacionado com o paciente. MAIA NETO $(1990$, p. 60) cita que “ médicos e enfermeiros entendem da atividade de atenção ao paciente; arquitetos, de espaço; o farmacêutico, de medicamentos; engenheiros, de estruturas e instalações; os economistas, de verbas; os administradores, de normas e conflitos, demonstrando assim claramente a necessidade de uma abordagem multidisciplinar e de uma linguagem comum." É imprescindível salientar que médicos e enfermeiros, além de entenderem da atividade de atenção ao paciente, cabe também a eles, o conhecimento de farmacologia para o exercício da sua atividade. $\mathrm{O}$ envolvimento multidisciplinar destes profissionais se traduz em benefício ao paciente e não na descaracterização do holístico.

No que tange à diminuição do tempo dispensado pela equipe de enfermagem e/ou enfermeiro na armazenagem e preparo da medicação, este tempo seria revertido para a assistência direta aos pacientes, permitindo uma atenção individualizada, mais humanizada, abrangendo as necessidades biopsicossociais e promovendo condições para a realização de novas pesquisas na enfermagem, o que incrementaria seu corpo de conhecimento. 
CONSIDERAÇÕES FINAIS

Finalizando estas reflexões, queremos destacar um ponto que consideramos crucial na implantação do SDMDU, que embora apareça como considerações finais, não é de menor importância. Este ponto é o dano ao paciente e mesmo a sua morte em decorrência de um medicamento com preparo inadequado ou errado.

Entendemos que isto possa ocorrer tanto no sistema tradicional de distribuição de medicamentos quanto no SDMDU. Quando acontece, no sistema tradicional, o enfermeiro e o auxiliar de enfermagem podem ser legalmente penalizados. Nesta condição o profissional terá consciência de seu erro, podendo refletir sobre seus atos, conhecimentos, habilidades e sua competência. Responderá por sua falha e a pena poderá proporcionar um melhor direcionamento na sua nova vida profissional.

A questão moral se complica quando o erro ocorre no SDMDU. Nesta circunstância o profissional será um mero agente provocador da morte. Entendemos neste caso, que ele não pode ser punido legalmente, já que o fármaco vem preparado. Porém, o sentimento de culpa por ter sido um instrumento de dano ou da morte é extremamente penoso.

Como trabalhar com estas situações quando ocorrem?

Como prevenir tais situações?

Parece-nos de suma importância refletirmos sobre estes aspectos e que nesta época de modernidade todo o empenho deve ser feito em benefício do paciente. Que as equipes interdisciplinares trabalhem cada vez mais coesas em prol do ser humano.

Acreditamos que a implantação do SDMDU sem um planejamento que abranja tanto os aspectos físicos e estruturais, quanto os de recursos humanos, poderá não atingir as metas pretendidas.

Para atividade de enfermagem este sistema é extremamente importante e sendo assim, o enfermeiro, reafirmamos, deve e precisa participar do processo de sua implantação.

\section{DISTRIBUTION SYSTEM OF ONLY-DOSE MEDICINES: REFLECTIONS ON NURSING PRACTICE}

The nurse, as a member of the staff, is supposed to take responsibility on the administration of medicines as one of the important duties among all the activities he/she has to perform. The implementation of the distribution system of only-dose medicine is directly bound to nurses' activities. Therefore, this study has as its main purpose to detect nurses' reflections about the system and their impact on nursing practice. The authors questioned the way the nurse and his/her staff are inserted in this context of changes, in a way of really assuring benefits for the patients, following and evaluating the whole process, considering that such change affects directly part of these activities.

KEY WORDS: only-dose, nursing care

\section{SISTEMA DE DISTRIBUCIÓN DE MEDICAMENTOS POR DOSIS UNITARIA: REFLEXIONES PARA LA PRÁCTICA DE ENFERMERÍA}

El enfermero, como parte integrante del equipo de salud, está obligado a conocer la responsabilidad que tiene en la administración de medicamentos como algo importante dentro del conjunto de actividades que realiza. La implantación del sistema de distribución de medicamentos por dosis unitaria se refleja directamente en las actividades de los enfermeros con relación a ese sistema y sus repercuciones en la práctica de la enfermería. Se cuestiona la forma de cómo lo enfermero y su equipo están insertados en este contexto de cámbios, en el sentido de que realmente asegure el beneficio para el paciente, acompañando y evaluando todo el proceso, ya que este cámbio afecta directamente una parte de su actividad.

TÉRMINOS CLAVES: dosis unitaria, asistencia de enfermería

\section{REFERÊNCIAS BIBLIOGRÁFICAS}

01. BRASIL. Ministério da Saúde. Coordenação de Controle de Infecção hospitalar. Guia básico para a farmácia hospitalar. Brasília: MS/SAS, 1994.

02. CONTROLE de infecção. Becton Dickinson, v. 2, n. 28, fev/abr. 1996.

03. MAIA NETO, J.F. Farmácia hospitalar: um enfoque sistêmico. Brasília: Thesaurus, 1990.
04. MENDONÇA, S.G.S. Dose unitária em pediatria - central de misturas endovenosas. São Paulo, 1996. mimeo.

05. PINERO, M.; MORGADO, C.; PEREZ, E. Importancia de la labor de la enfermeira en la administration de medicamentos por dosis unitária. Rev. Cuba. Enfermagem, v. 2, n. 2, p. 107-115, maio/ago. 1986.

06. SANTOS, G.A.A. Consumo de líquidos, antes/ depois da implantação da dose unitária. São Paulo, 1996. mimeo. 


\section{BIBLIOGRAFIA CONSULTADA}

01. BARBOSA, E.M. A farmácia nas ações de combate à infecção hospitalar na aeronáutica. Rev. Méd. Aeronaut. Bras., v. 41, n. 1, p. 722, jan/jul. 1991.
02. CONSELHO REGIONAL DE ENFERMAGEM. Principais legislações para o exercício da enfermagem. São Paulo, 1996.

03. RIBEIRO, E. Dose unitária: sistema de distribuição de medicamentos em hospitais. São Paulo, 1128p. Dissertação (Mestrado) - Escola de Administração de Empresas de São Paulo, 1991. 